\title{
Deskilling in ophthalmology is the inevitable controllable?
}

\author{
Jaime Levy $\mathbb{1}^{1} \cdot$ Alan Jotkowitz $^{2} \cdot$ Itay Chowers ${ }^{1}$
}

Received: 28 August 2018 / Accepted: 6 September 2018 / Published online: 24 October 2018

(c) The Royal College of Ophthalmologists 2018

\section{The evolving art of medicine and the "problem" with technology}

Medicine is an ever-changing art. The rate of change and its consequences bare important implications to patients and physicians alike. Ophthalmology is not an exception in that sense. Up until around the turn of the Millennium, an ophthalmologist had to develop diagnostic skills which were heavily dependent upon one's ability to identify correctly pathological findings. The ophthalmologist had then to determine the diagnosis and develop a treatment plan. These characteristics of the profession were similar in developed or developing countries. While major differences among such countries were availability of professionals and treatment equipment.

During the last few years, we have witnessed the increasing use of technology in the field of ophthalmology. Advances such as optical coherence tomography (OCT) and OCT-angiography, as well as sophisticated biometry and cornea photography, have and are revolutionizing daily practice in ophthalmology clinics worldwide.

For example, OCT surrogate end-points and biomarkers are displacing other historical measurements such as visual acuity and clinical examination in clinical trials and it routine practice. The use of this new technology has busted the diagnostic accuracy of retinal diseases, but, it also created a whole host of new challenges, such as data storage, interpretation, learning, cost and maintenance. An additional problem is the fact that ophthalmologists in training who begin to examine patients and learn various pathologies, may be prone to diagnose, treat or

$\triangle$ Jaime Levy

levjaime@gmail.com

1 Department of Ophthalmology, Hadassah Medical Center, and the Hebrew University-Hadassah School of Medicine,

Jerusalem, Israel

2 Department of Internal Medicine, Faculty of Health Sciences, BenGurion University of the Negev, Beer-Sheva, Israel make decisions based solely on OCT and similar sophisticated technologies even without thoroughly examining the patient. In a short time, the "physical examination" of the patient can pass to the background or even to the dustbin history.

\section{Deep learning}

Deep learning algorithms applied to the field of the ophthalmology are bearing fruit. In the field of retina, deep learning builds upon the data generated by modern imaging modalities. Research on automatic detection of diabetes retinopathy, age-related macular degeneration (AMD), glaucoma, decision on whether to inject or not an eye with neovascular AMD, and many more, are appearing in the ophthalmological literature in recent years. The first system which autonomously screens diabetic patients for retinopathy was recently approved by the FDA. In other fields of medicine, such as radiology and pathology, which bear much resemblance to ophthalmology in which the diagnosis is visual and a large number of images must be reviewed to see if there is for example, a pulmonary embolus on computed tomography, an abnormal cell on the histological slide on minimal subretinal fluid on OCT, voices are emerging that advise specialists to become an "information specialist" to captain artificial intelligence and provide a better medical service [1].

\section{Deskilling}

The origin of the term deskilling comes from Marx in the 19th century and the escalating labor force in factories, the decline of craft skills and its replacement with technological means [2]. Deskilling is the reduction of the level of skill required to complete a task when some or all components of the task are partly automated [3]. This process can affect physicians' ability to derive informed opinions on the basis of detectable signs, symptoms, and available data. Deskilling itself is rarely a problem; however, it becomes one if the technology fails or 
breaks down and if the sociotechnical system relies on the failed technology to complete the work [3].

\section{Residents}

Deskilling gained little attention in the ophthalmology field. Primary care physicians using two-work innovation, such as electronic medical records and clinical guidelines, experienced deskilling [4]. The deskilling outcomes identified include decreased clinical knowledge, decreased patient trust, increased stereotyping of patients, and decreased confidence in making clinical decisions. Physical examination performed by surgical residents is inaccurate in identifying significant pathologies in penetrating thoracic trauma and examination skill may have deteriorated over the years. Reliance on technology and laboratory tests, inadequate teaching, reduction in bedside time and direct physician-patient interaction, and even failure to perform a complete physical examination, have been all reported to explain in part the loss of physical examination skill by internal medicine students and residents [5].

Ocular fundus photographs may be better interpreted than direct ophthalmoscopy by non-ophthalmologist emergency care providers [6]. Surgical competencies of ophthalmology residents are continuously studied and validation efforts examined. However, non-surgical clinical skills are encompassed together with clinical imaging and technologies since the beginning of the residency. Accurate interpretation of ophthalmic imaging is promptly required and diagnosis based on retinal OCT may be much faster and easy than a carful fundus examination.

Ophthalmology residents in developing countries have less access to state-of-the-art technologies. International graduates in industrialized countries who have not been strongly taught on physical examination coming back to their native areas without imaging devices, will be of much less value than a well-trained ophthalmology resident who has sill independently on the technology. On the other hand, ophthalmologist trained in a program characterized by lagging technology may have further difficulty in practicing at an updated technology environment. Thereby, a larger professional rift is generated across countries and regions.

Both senior and young ophthalmologists may be affected by the deskilling process, however, residents may be more sensitive. Rapid evolution of ophthalmic technology has been linked with high rates of ophthalmology residents burnout [7]. Thus, program directors and their departments are advised to develop, strengthen and evaluate resident wellness programs.

\section{Strategies for prevention}

Technology is invaluable in ophthalmology but not infallible. We should still be able to perform a complete ophthalmic physical examination and to teach adequately this art to our medical students and residents. As Donald Gass described the stages and pathogenesis of macular holes 30 years ago without the aid of an OCT device, we might better understand new clinical challenges if we rely on physical examination first and then confront the findings with all available high-tech outputs. It is not too late for Ophthalmology to retain the art of clinical examination. A core of skills that should not be allowed to "deskilled" such as detailed ophthalmoscopy, and identification of corneal pathology should be defined and incorporated to the residency syllabus regardless of technological advancement. While these tasks seem trivial today, history has taught us how swift a skill may vanish. Just think of the number of extracapsular cataract extraction procedures performed by a resident in an Ophthalmology Department in a developed country 25 years ago and the average number of procedures the resident performs during the entire residency at present (2.2 cases in 2014-2015 according to Accreditation Council for Graduate Medical Education ophthalmology resident surgical case logs). Consequently residents may permanently lose surgical skills in cases of complicated cataract and suturing of corneal lacerations.

\section{Compliance with ethical standards}

Conflict of interest The authors declare that they have no conflict of interest.

\section{References}

1. Jha S, Topol EJ. Adapting to artificial intelligence: radiologists and pathologists as information specialists. JAMA. 2016;316:2353-4.

2. Fernandez-Castrillo C. Rethinking interdisciplinarity, In: Adamowicz E, Storchi S, editors. Back to the futurists. The avant-garde and its legacy. Manchester University Press. Manchester. 2013; p. 286.

3. Cabitza F, Rasoini R, Gensini GF. Unintended consequences of machine learning in medicine. JAMA. 2017;318:517-8.

4. Hoff T. Deskilling and adaptation among primary care physicians using two work innovations. Health Care Manag Rev. 2011;36:338-48.

5. Verghese A, Charlton B, Kassirer JP, Ramsey M, Ioannidis JP. Inadequacies of physical examination as a cause of medical errors and adverse events: a collection of vignettes. Am J Med. 2015;128:1322-4. e3

6. Biousse V, Bruce BB, Newman NJ. Ophthalmoscopy in the 21st century: The 2017 H. Houston Merritt Lecture. Neurology. 2018;90:167-75.

7. Sadun AA. The Challenge of teaching ophthalmology: a residency review committee perspective. Ophthalmology. 2000;107:1971-2. 